\title{
Unveiling the Crucial Role of Impact nergy Loss for So-Called Incompressible Fluid Flow
}

\author{
Jiunn-Shean Chiang \\ Graduate Student of Mechanical Engineering, National Chung-Hsing University, Taichung, Taiwan 402, R.O.C. \\ Shu-Hao Chuang \\ Professor of Mechanical Engineering, National Chung-Hsing University, Taichung, Taiwan 402, R.O.C. \\ Hsing-Juin Lee \\ Professor of Mechanical Engineering, National Chung-Hsing University, Taichung, Taiwan 402, R.O.C., \\ microprop@yammail.com
}

Follow this and additional works at: https://jmstt.ntou.edu.tw/journal

Part of the Mechanical Engineering Commons

\section{Recommended Citation}

Chiang, Jiunn-Shean; Chuang, Shu-Hao; and Lee, Hsing-Juin (2004) "Unveiling the Crucial Role of Impact nergy Loss for So-Called Incompressible Fluid Flow," Journal of Marine Science and Technology. Vol. 12: Iss. 1, Article 6. DOI: $10.51400 / 2709-6998.2219$

Available at: https://jmstt.ntou.edu.tw/journal/vol12/iss1/6

This Research Article is brought to you for free and open access by Journal of Marine Science and Technology. It has been accepted for inclusion in Journal of Marine Science and Technology by an authorized editor of Journal of Marine Science and Technology. 


\title{
UNVEILING THE CRUCIAL ROLE OF IMPACT ENERGY LOSS FOR SO-CALLED INCOMPRESSIBLE FLUID FLOW
}

\author{
Jiunn-Shean Chiang*, Shu-Hao Chuang**, and Hsing-Juin Lee**
}

Key words: incompressible pipe expansion flow, impact energy loss, unveiling crucial role, triggering FD/CFD revolution.

\begin{abstract}
Experiments show that pipe flows with large expansion angle and area ratio always accompany with significant amount of energy loss, actually energy loss ratios over $70 \%$ of incoming flow kinetic energy are commonplace. It is generally believed that all the energy loss is entirely due to viscous dissipation effect. In this paper, several interesting demonstrations have been given to prove the existence and importance of impact energy loss. Literally, impact energy loss is usually more important than viscous energy loss in many cases. In fact, the pipe expansion flow can be configured as high-speed flow ramming into low-speed flow and then moving together, this situation is similar to rope-lifting problem in system dynamics as illustrated herein as continuous inelastic impact process with unavoidable impact energy loss. Virtually the intrinsic negligence of impact energy loss is the original major cause for many weird paradoxes and disaster solutions in fluid dynamics. Moreover, in CFD practice, many kinds of ad hoc treatments such as artificial viscosity, numerical dissipation, upwinding and so forth are devised awkwardly in an attempt to somehow manipulate a certain amount of disguised energy dissipation that is largely due to impact. These treatments are done as usual without recognizing what an important role the impact energy loss is playing in the game. More surprisingly, this intrinsic negligence of impact energy loss in deriving process renders the Euler equation as a non-genuine momentum equation which is hardly solvable, and in turn unveils the even more stunning role of impact energy loss as the triggering concept for a full-scale historical revolution in FD/CFD as deliberated in the related paper [10].
\end{abstract}

\section{INTRODUCTION}

The transport processes of fluid flow in piping system with expansions are substantially important engineering problems that are frequently encountered. A general survey shows that pipe flows with large expansion angle and area ratio always accompany with sig-

Paper Submitted 10/09/03, Accepted 12/26/03. Author for Correspondence: Hsing-Juin Lee. E-mail: microprop@yammail.com.

*Graduate Student of Mechanical Engineering, National Chung-Hsing University, Taichung, Taiwan 402, R.O.C.

**Professor of Mechanical Engineering, National Chung-Hsing University, Taichung, Taiwan 402, R.O.C. nificant amount of energy loss as evidenced in Figs. 1 and 2 for gradual pipe expansion flows. Sometimes the energy loss ratio can be easily over $70 \%$ of incoming flow kinetic energy, as shown in Figs. 3 and 4 for sudden pipe expansion flows. But what causes it? It is generally believed that all the energy loss involved in fluid flowing is entirely due to viscous dissipation effect. Nevertheless, in the following sections, no matter how surprisingly, we will unveil the impact energy loss as the true major culprit for most energy loss in many gradual and sudden pipe expansion flows. And more surprisingly, this concept of impact energy loss will subsequently trigger a full-scale revolution in the paradoxes-rotten fundamental theoretical system of fluid dynamics, and associated computational fluid dynamics. This all-out revolution in FD/CFD will be only broadbrushed in this paper, its more theoretical aspects will be deliberated in a sequential paper [10] titled "The Life and Death of Euler, Bernoulli, Navier-Stokes Equations and Associated CFD for So-called Incompressible Fluid Flow". Then we can see what a crucial role the impact energy loss is playing in the FD/CFD field. Very interestingly, all these landslides in FD/ CFD can be attributed to a corresponding absurdity of the rope-lifting problem in system dynamics also due to disregarding impact energy loss as illustrated in later section.

\section{CONCEPT OF IMPACT ENERGY LOSS IN FLUID DYNAMICS}

Fluid mechanics analysis is naturally based on the conservation laws of mass, momentum and energy. In general, water as the most common fluid is considered to be incompressible, but in reality water is slightly compressible with a high bulk modulus and extremely low viscosity. For example, it would take a high pressure of $212 \times 10^{5} \mathrm{~N} / \mathrm{m}^{2}$ (about 3,120 psi) to compress $1 \%$ of water by volume [15], yet under the condition of one atmosphere pressure and temperature of $16^{\circ} \mathrm{C}$ or so, the kinematic viscosity of water is only $1.12 \times 10^{-6} \mathrm{~m}^{2} / \mathrm{s}$. But with such a minimum viscosity, what truly causes 
such a sizable energy loss in the pipe flow with large expansion angle and area ratio? Well, people may argue that the energy loss in such pipe expansion flows is all due to the fluid viscosity. In fact, with very short duration for water flowing through a short length of expansion portion of pipe, there is essentially no significant chance for viscous effect to rip off such a high percentage of incoming flow kinetic energy. Alter-natively, pipe expansion flow can be configured as high-speed flow ramming into low-speed flow and then moving together, this situation is similar to the continuous inelastic impact process in system dynamics to be illus-

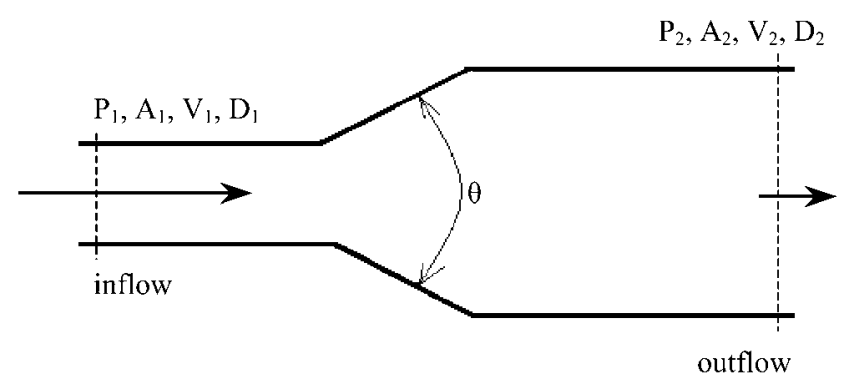

Fig. 1. The configuration of gradual pipe expansion flow.

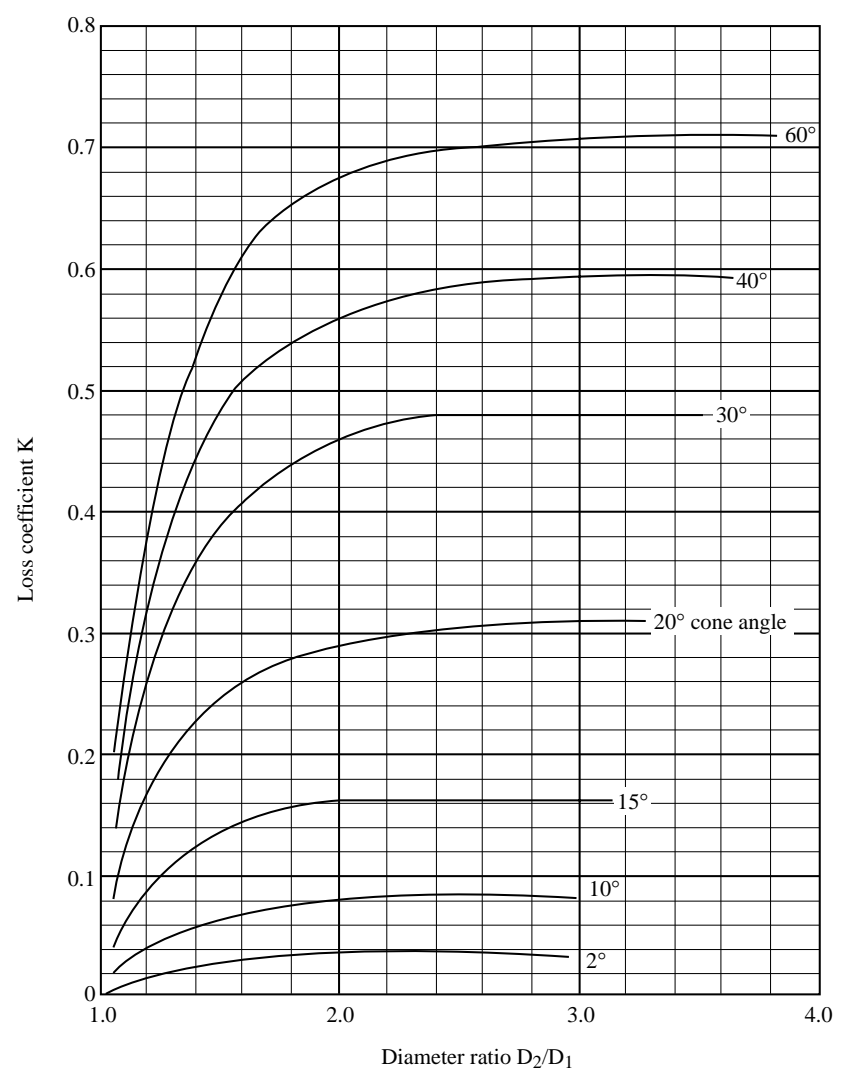

Fig. 2. The loss coefficient for gradual pipe expansion flow [4]. trated later. Notably, this kind of dynamics process involves unavoidable impact energy loss as well known.

In order to prove the important idea that even with little chance for viscous dissipation, there still could be considerable amount of energy loss due to impact. Let us consider an interesting experiment: when two blocks of viscous and so-called incompressible water flow collide against each other in a straight pipe, what would happen after this impacting ? The result is that most of

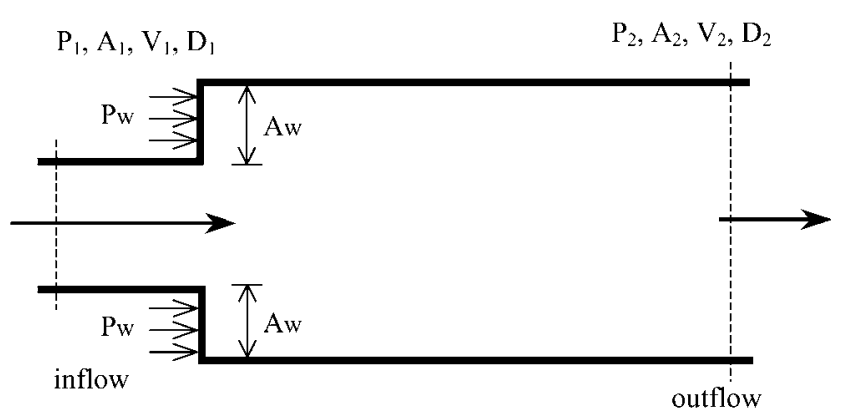

Fig. 3. The configuration of sudden pipe expansion flow.

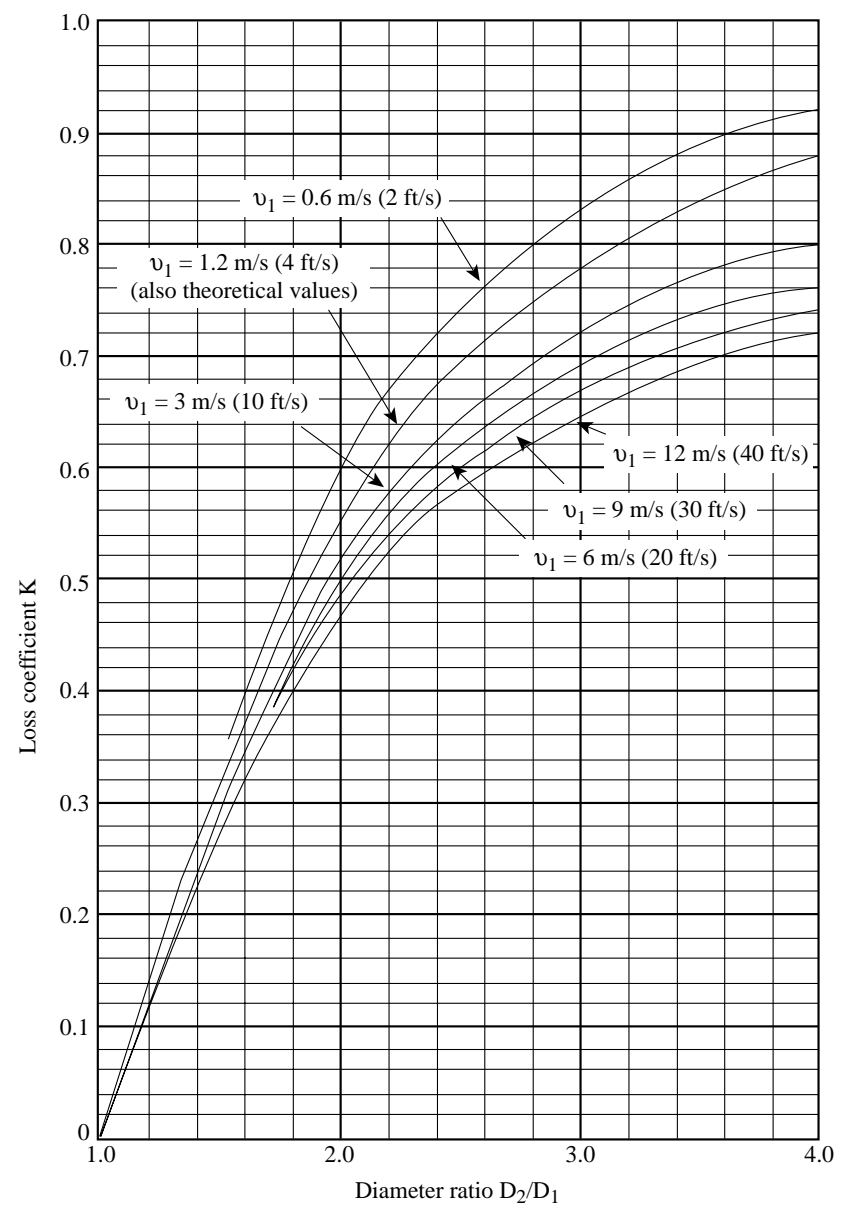

Fig. 4. The loss coefficient for sudden pipe expansion flow [4]. 
the kinetic energy was dissipated during this impact process, yet the nearly paralleled flow has very slim chance to activate the viscous energy dissipation. Such a striking example has clearly illustrated the viscous energy loss plays a very limited role for energy dissipation compared with impact energy loss which can be preliminarily thought as the fluid flow energy loss in addition to the usual viscous energy loss. Further, numerous daily phenomena such as falling rain on the ground, water hammer, hydraulic jump, water flow into reservoir and so forth, can literally demonstrate the existence and importance of impact energy loss in fluid flow. Virtually, in decreasing viscosity experiment by measuring the drag of fluid flow past a circular cylinder, we would find that no matter how small one makes the viscosity, one would still measure a finite drag, essentially independent of the value of viscosity [15].

Moreover, to our knowledge, no literature shows any significant difference for energy loss of pipe flow with large expansion angle and area ratio, even when $90^{\circ} \mathrm{C}$ water with a kinetic viscosity of $3.2 \times 10^{-7} \mathrm{~m}^{2} / \mathrm{s}$ is replaced by $20^{\circ} \mathrm{C}$ water with a kinetic viscosity of 10.0 $\times 10^{-7} \mathrm{~m}^{2} / \mathrm{s}$ as shown in Table 1 (which is more than $300 \%$ of the $90^{\circ} \mathrm{C}$ water viscosity [17]). This vivid example of cold/hot water pipe expansion flow has further clarified the existence/importance of impact energy loss. Besides, the amount of impact energy loss in the pipe expansion flowing process depends on a spectrum of physical parameters, including the expansion angle, expansion curve, the area ratio, the inflow velocity, the physical parameters of flowing media, and etc. In light of its complexity, it is understandable so far most energy loss data for pipe expansion flows are experimental.

Table 1. Water viscosity vs. temperature [2]

\begin{tabular}{|c|c|c|c|}
\hline Temperature & Density & $\begin{array}{l}\text { Dynamic } \\
\text { Viscosity }\end{array}$ & $\begin{array}{l}\text { Kinematic } \\
\text { Viscosity }\end{array}$ \\
\hline $\begin{array}{c}\mathrm{T} \\
\left({ }^{\circ} \mathrm{C}\right)\end{array}$ & $\begin{array}{c}\rho \\
\left(\mathrm{Kg} / \mathrm{m}^{3}\right)\end{array}$ & $\begin{array}{c}\mu \\
\left(\mathrm{N} \cdot \mathrm{s} / \mathrm{m}^{2}\right)\end{array}$ & $\begin{array}{c}v \\
\left(\mathrm{~m}^{2} / \mathrm{s}\right)\end{array}$ \\
\hline 000 & 999.9 & $1.787 \mathrm{E}-3$ & 1.787 E-6 \\
\hline 005 & 1000.0 & $1.519 \mathrm{E}-3$ & 1.519 E-6 \\
\hline 010 & 999.7 & $1.307 \mathrm{E}-3$ & 1.307 E-6 \\
\hline 020 & 998.2 & $1.002 \mathrm{E}-3$ & 1.004 E-6 \\
\hline 030 & 995.7 & $7.975 \mathrm{E}-4$ & 8.009 E-7 \\
\hline 040 & 992.2 & $6.529 \mathrm{E}-4$ & $6.580 \mathrm{E}-7$ \\
\hline 050 & 988.1 & $5.468 \mathrm{E}-4$ & 5.534 E-7 \\
\hline 060 & 983.2 & $4.665 \mathrm{E}-4$ & 4.745 E-7 \\
\hline 070 & 977.8 & $4.042 \mathrm{E}-4$ & 4.134 E-7 \\
\hline 080 & 971.8 & $3.547 \mathrm{E}-4$ & $3.650 \mathrm{E}-7$ \\
\hline 090 & 965.3 & $3.147 \mathrm{E}-4$ & $3.260 \mathrm{E}-7$ \\
\hline 100 & 958.4 & $2.818 \mathrm{E}-4$ & $2.940 \mathrm{E}-7$ \\
\hline
\end{tabular}

Furthermore, to have some real-life feeling about quantitatively how important the impact energy loss is, let us take the pipe flow with sudden expansion as an example, if for diameter ratio of 3.6, the experimental data show that $90 \%$ of incoming flow kinetic energy could be lost as seen in Fig. 4 [14]. With above understanding, now it can be rightfully recognized the major culprit for such a sizable energy loss for pipe flow with large expansion angle and area ratio is actually the impact energy loss, which is usually much more important than viscous energy loss in many flowing situations. No wonder distinguished scholar like Munson et al. [15] ever indicated that as with so many energy loss situations, it is not the viscous effects directly that cause the loss, rather, it is the dissipation of kinetic energy as the fluid decelerates inefficiently. Further, let us quote Lomax [12] that "Although numerical approximation to the Navier-Stokes equations contain dissipation through the viscous terms, this can be insufficient, especially at high Reynolds numbers". And more sarcastically, pioneering researcher Ladyzhenskaya [9] once expressed that in many flow cases, viscosity only serves as the scapegoat for answering many accumulated absurdities in the theory of fluid dynamics. In fact, their common hunches $[1,3-7,9,12,15,18-20]$ are in good accordance with the crucial concept of impact energy loss to be unveiled here for so-called incompressible fluid flows.

Besides, for potential flow as incompressible, inviscid, irrotational, and steady fluid flow, we will see that the intrinsic negligence of impact energy loss is also the major cause for many weird paradoxes [2] and disaster solutions such as d'Alembert's-the unrealistic and weird phenomenon of zero drag force resulting from uniform fluid flow past a circular cylinder as shown in Fig. 5. It is interesting to note that, geometri-

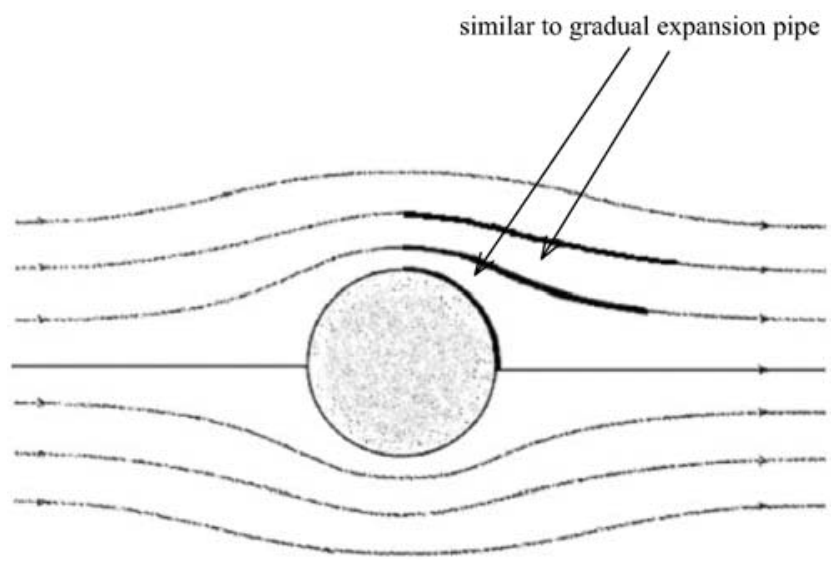

Fig. 5. The general streamline pattern of potential flow for uniform flow past a circular cylinder [3]. 
cally this paradox is not a paradox that "full", since for the frontal portion within $\pm 60^{\circ}$ angle or so, the potential flow theory still gives an acceptable prediction of pressure distribution in dimensionless form compared with experimental data as shown in Fig. 6 (where $P_{0}$ is the general far-field pressure and $P_{S}$ is the surface pressure). Yet, the ensuing portion $\left(>60^{\circ}\right)$ fails miserably to reduce pressure largely due to disregarding the crucial impact energy loss with its expansion flow.

\section{IMPACT ENERGY LOSS-THE TRIGGERING CONCEPT FOR FD/CFD REVOLUTION}

The Euler equation along streamtube for seemingly incompressible inviscid flow with cross-section A can be expressed as

$$
-(d P) A=\rho A V d V
$$

where $\rho$ is the density, $V$ is the flowing velocity and $P$ is the streamwise pressure. Note that gravity term is not written in for the sake of simplicity. Then it can be integrated along streamtube to become the famous Bernoulli equation.

$$
\frac{V_{1}^{2}}{2 g}+\frac{P_{1}}{\gamma}=\frac{V_{2}^{2}}{2 g}+\frac{P_{2}}{\gamma}
$$

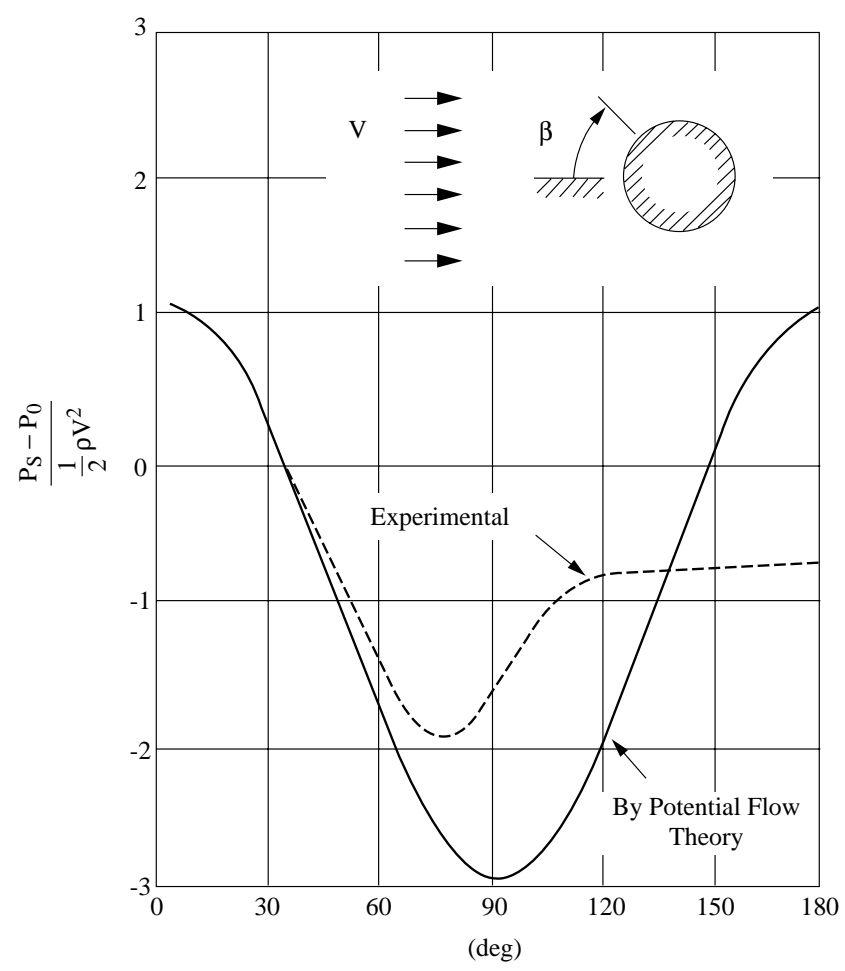

Fig. 6. The pressure distribution in dimensionless form on the surface of a circular cylinder for uniform incoming flow [2]. where $g$ is the local gravity acceleration, $\gamma$ is the weight density. However, it is well-known that Euler equation as represented by Bernoulli equation can only accommodate no-energy-loss cases, yet they cannot accommodate any cases with energy loss at all. Although they are derived by momentum principle, no matter how surprisingly, they are not genuine momentum equations, since a genuine momentum equation should be able to accommodate both cases either with or without energy loss [10].

Subsequently, for the sake of seeing some insights of the above problem, let us take the sudden pipe expansion flow shown in Fig. 3 as an example. Then, the associated continuity, momentum and energy equations with possible head loss $h_{L}$ can be sequentially expressed as

$$
\begin{aligned}
& A_{1} V_{1}=A_{2} V_{2}=A V \\
& P_{1} A_{1}-P_{2} A_{2}+P_{W} A_{W}=\rho A V\left(V_{2}-V_{1}\right) \\
& \frac{P_{1}}{\gamma}+\frac{V_{1}^{2}}{2 g}=\frac{P_{2}}{\gamma}+\frac{V_{2}^{2}}{2 g}+h_{L}
\end{aligned}
$$

where $P_{W}$ is the wall pressure and $A_{W}$ is the pipe wall area as shown in Fig. 3. From the momentum Eq. (4) for sudden pipe expansion flow, we clearly see that the $P_{W}$ acting on $A_{W}$ can be adjusted in accordance with $P_{2}$, so that they can always satisfy the momentum equation and accommodate any energy loss induced during this flowing process. Consequently, for known $A_{1}, A_{2}, P_{1}, V_{1}$ the solution for the system is not only existent but also unique if $h_{L}, P_{2}$, or $P_{W}$ is given. For example, as illustrated by Munson et al. [15] and others, if one assumes $P_{W}=0$, then Eqs. (3) (5) can be simultaneously solved to obtain the head loss.

$$
h_{L}=\left(1-\frac{A_{1}}{A_{2}}\right)^{2} \frac{V_{1}^{2}}{2 g}=K \frac{V_{1}^{2}}{2 g}
$$

where the loss coefficient $K$ [15] stands for the energy loss ratio compared with the incoming flow kinetic energy. To have some numerical feeling, if taking area ratio $A_{2} / A_{1}=(2.4)^{2}=5.76$ as an example, then the result $K=0.683$ agrees well with the experimental data shown in Fig. 4 [14].

On the other hand, even when experimental data $A_{1}, A_{2}, P_{1}, V_{1}$ and the outflow pressure $P_{2}$ are given, the solution of Euler equation may not exist at all due to its incapability of accommodating any energy loss in order to reduce the outflow pressure to the realistic level which is usually lower than what Euler equation can predict for general pipe expansion flows. That is the reason why the seemingly simple-looking Euler equation for incompressible, inviscid flow constitutes one of 
the most difficult problems in FD/CFD to be solved, because the solution is not existent at all for many cases if disregarding impact energy loss. No wonder, in computational fluid dynamics practice, many kinds of sophisticated ad hoc treatments such as artificial viscosity, numerical dissipation, upwinding, and etc., are devised awkwardly in an attempt to somehow manipulate a certain amount of disguised energy dissipation which is largely due to impact.

Besides the straightforward adding of artificial viscosity, other artificial measures such as the popular upwind method [16] are just involving the artificial viscosity in a seemingly more innocent manner. For instance, the upwinding scheme for one-sided difference method [8] can be expressed as

$$
\frac{d u}{d s} \cong \frac{u_{i}-u_{i-1}}{h}=\frac{u_{i+1}-u_{i-1}}{2 h}-\frac{h}{2}\left(\frac{u_{i+1}-2 u_{i}+u_{i-1}}{h^{2}}\right)
$$

where $u$ is the flow velocity, $s$ is the streamwise coordinate, and $h$ is the grid space. By careful observation, the first term on the right-hand side is the first-order central difference approximation. The last parentheses represent the central difference approximation of the second order derivative.

$$
\begin{aligned}
\frac{d^{2} u}{d s^{2}}=\frac{d}{d s}\left(\frac{d u}{d s}\right) & \cong\left[\frac{1}{h}\left(\frac{u_{i+1}-u_{i}}{h}-\frac{u_{i}-u_{i-1}}{h}\right)\right] \\
& =\frac{u_{i+1}-2 u_{i}+u_{i-1}}{h^{2}}
\end{aligned}
$$

While the coefficient $h / 2$ in front of the right-hand parentheses in Eq. (7) is equivalent to the dynamic viscosity coefficient $\mu$, we can clearly see that the

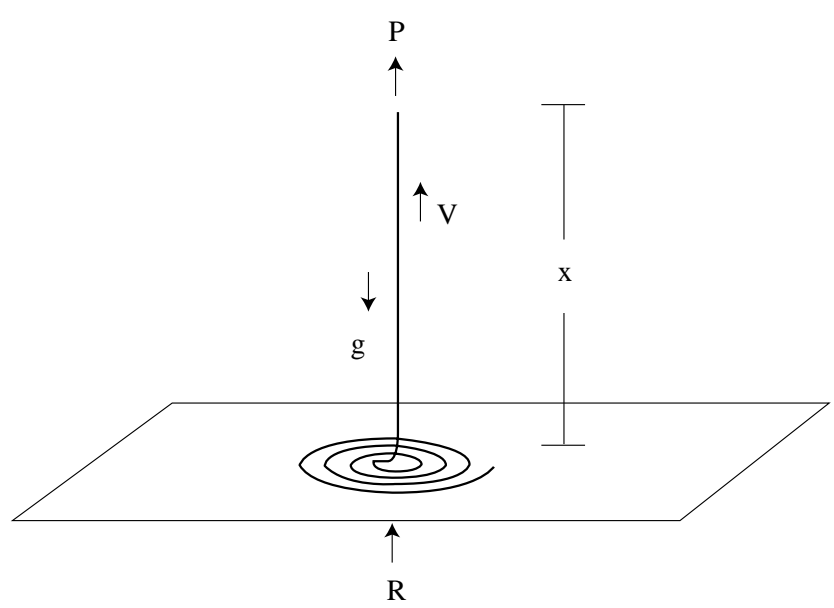

Fig. 7. The rope-lifting problem with constant pulling velocity $V$. upwind method is just artificial viscosity in different form to play an energy dissipation role in CFD. This method is managing to somehow cover the impact energy loss in a disguised manner in order to stand a better chance for acquiring more reasonable solutions than the central-difference scheme in many real flow cases. No wonder, some highly seasoned scholars ever indicate that CFD is still very much an art than a science $[3,7]$.

Now we know that the intrinsic negligence of impact energy loss in Euler equations results in their non-genuineness as momentum equations and thus constitutes endless troubles in solving incompressible flow [10]. Eventually, we can recognize that the impact energy loss is just the triggering concept for future FD/ CFD revolution as deliberated in a relevant paper [10]. Very interestingly, this full-scale revolution in FD/CFD can be originated from our finding of a seemingly unrelated absurdity in the system dynamics concerning a rope-lifting problem as illustrated in the following section.

\section{THE SLIPPERY ROPE-LIFTING PROBLEM OF SYSTEM DYNAMICS}

Therefore, let us further examine a corresponding example in system dynamics and see what a strange conclusion can scientists reach if disregarding impact energy loss: When we lift a flexible and inextensible rope piled on ground as shown in Fig. 7. Traditionally, this problem may be tentatively solved by applying the work-energy equation to the entire rope for an infinitesimal movement $\mathrm{dx}$ [13]

$$
P d x=d\left(\frac{1}{2} \rho x V^{2}\right)+d\left(\rho g x \frac{x}{2}\right)
$$

or

$$
P d x=\frac{1}{2}(\rho d x) V^{2}+(\rho g x) d x
$$

or simply

$$
P=\frac{1}{2} \rho V^{2}+\rho g x
$$

where $P$ is the pulling force applied at the upper end of rope to elevate it, $\rho$ is the mass density per unit length of rope, $x$ is the length of the portion of rope already pulled up, and $V$ is the constant pulling velocity. Noting that in the above equations, the work done by the lifting force $P$ becomes the kinetic energy and gravitational potential energy with the implicit assumption of no energy loss. Next, the force-momentum equation can be applied to the entire rope system

$$
P+R-\rho g L=\frac{d}{d t}(\rho x V)
$$


or

$$
P+R=\rho g L+\rho V^{2}
$$

where $R$ is the ground reaction force and $L$ is the total length of the rope. Eliminating $P$ between Eqs. (11) and (13), one can obtain

$$
R=\rho g L+\rho V^{2}-\left(\frac{1}{2} \rho V^{2}+\rho g x\right)
$$

or simply

$$
R=\rho g(L-x)+\frac{1}{2} \rho V^{2}
$$

When at the beginning of pulling, one can substitute $x=0$ into Eq. (15)

$$
\begin{aligned}
R & =\rho g(L-0)+\frac{1}{2} \rho V^{2} \\
& =W+\frac{1}{2} \rho V^{2}
\end{aligned}
$$

where $W$ is the weight of the entire rope. This bizarre solution of Eq. (16) indicates that if one puts a flexible and inextensible rope on a weighing scale, then pulling it upward, it will weight heavier than its weight at that instant. Alas! What a strange solution against common sense of physics! More incredibly, Eq. (16) says the faster one pulls the rope upward, the heavier it will show on the scale. Through above derivation with implicit no-energy-loss assumption for this dynamics process, it turns out to be such a peculiar result Eq. (16) with obvious irrationality. It is not so easy to find the culprit for this strange result until one looks at the problem with the viewpoint of continuous perfectly inelastic impact [11]. In that light, an interesting perfectly inelastic impact model with one-way locking mechanism as shown in Figs. 8 and 9 is devised to explain the crucial energy transformation phenomenon in such a system dynamics process.

First, the perfectly inelastic impact dynamics is briefly reviewed with masses $m_{1}$ and $m_{2}$ shown in Figs. 8 and 9. The $m_{2}$ is equipped with a special frictionless one-way locking mechanism to allow relatively approaching velocity and to avoid any relative separating velocity. The linear momentum equation can be expressed as

$$
m_{1} V_{1}=\left(m_{1}+m_{2}\right) V_{f}
$$

where $V_{f}$ is the common velocity of both $m_{1}$ and $m_{2}$ after the impact. Thus

$$
V_{f}=\left(\frac{m_{1}}{m_{1}+m_{2}}\right) V_{1}
$$

The eventual result after impact as illustrated in Fig. 9 is a typical perfectly inelastic impact process. Energy is partly transformed into spring energy in the impact process. Then, the remained kinetic energy $E_{k}$ for $m_{1}$ and $m_{2}$ together after the impact is

$$
\begin{aligned}
E_{k} & =\frac{1}{2}\left(m_{1}+m_{2}\right) V_{f}^{2} \\
& =\frac{m_{1}}{m_{1}+m_{2}}\left(\frac{1}{2} m_{1} V_{1}^{2}\right)
\end{aligned}
$$

Also, the energy transformed into spring energy during impact process is

$$
\begin{aligned}
E_{t f} & =\frac{1}{2} m_{1} V_{1}^{2}-E_{K} \\
& =\frac{m_{2}}{m_{1}+m_{2}}\left(\frac{1}{2} m_{1} V_{1}^{2}\right)
\end{aligned}
$$

The rope-lifting problem can be regarded as an infinitesimal portion of rope $d x$ remaining stationary just before being pulled upward to accelerate to the constant velocity of the upper portion of the rope. The situation is similar to the perfectly inelastic impact example for $m_{1}$ and $m_{2}$ [11] in Figs. 8 and 9. The $E_{k}$ acquired by $\rho d x$ is clearly equal to $\frac{1}{2}(\rho d x) V^{2}$ after being

$\mathrm{m}_{2}$

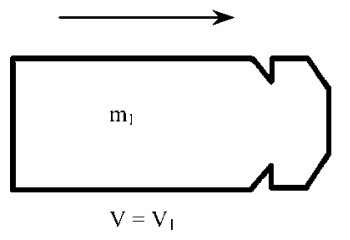

WWMY

$\mathrm{V}=0$

Fig. 8. Perfectly inelastic impact energy loss model before impacting.

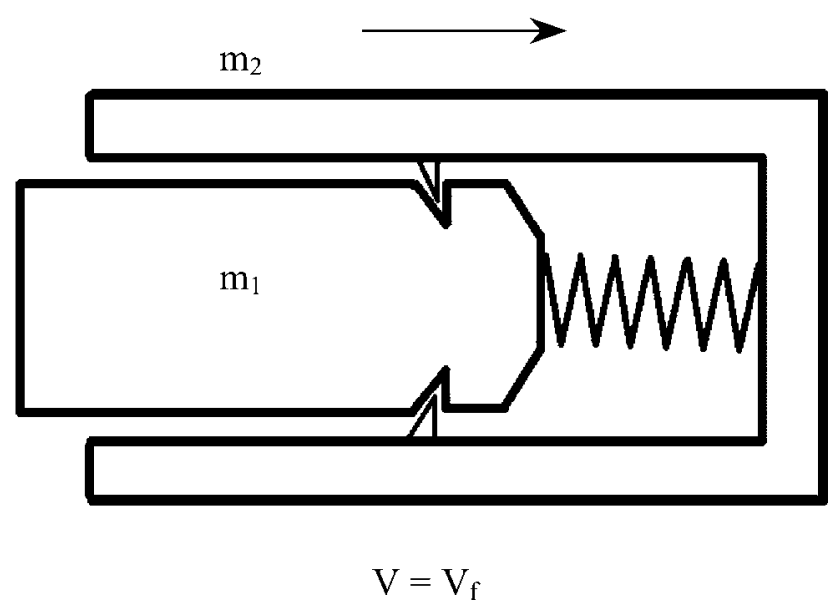

Fig. 9. Perfectly inelastic impact energy loss model with comoving velocity $V_{f}$ after impacting. 
pulled up. Corresponding to Eq. (20), the transformed energy for the upper portion of the rope is

$$
\begin{aligned}
E_{t f} & =\frac{\rho d x}{\rho x+\rho d x}\left(\frac{1}{2} \rho x V^{2}\right) \\
& =\frac{1}{2}(\rho d x) V^{2}
\end{aligned}
$$

For the rope system, the work input $P d x$ should be equal to all the energy increase of the rope system, including kinetic energy increase, transformed energy increase and gravitational potential energy increase

$$
\begin{aligned}
P d x & =d E_{K}+d E_{t f}+d E_{P} \\
& =\frac{1}{2}(\rho d x) V^{2}+\frac{1}{2}(\rho d x) V^{2}+(\rho g x) d x
\end{aligned}
$$

Thus

$$
P=\rho V^{2}+\rho g x
$$

Eq. (23) is clearly different from Eq. (11) which is incorrect due to the missing of the transformed energy term. Together with Eq. (13), the correct and natural reactive force $R$ can be thus obtained as a check

$$
R=\rho g(L-x)
$$

The main theme here is that, even for an implicit perfectly inelastic impact process, there must be always a certain amount of impact energy being lost as shown in Eq. (21). Consequently, any assertion of no energy loss in a perfectly inelastic impact process will give erroneous and strange result as in Eq. (15). No matter explicit or not, the original assumption of no energy loss is the fatal cause that makes the result of Eq. (15) appear so strange. In like manner, high speed flow ramming into low speed flow in pipe expansion case is similar to the continuous inelastic impact process in system dynamics with unavoidable impact energy loss. Correspondingly, strange results will likewise occur in incompressible fluid flow problems if disregarding impact energy loss. This striking example neatly unveils the existence and importance of the impact energy loss not only in fluid dynamics but also in related system dynamics. Although this standard rope-lifting problem is widely taught in many universities throughout the world, can any one believe this kind of absurdity shown in Eq. (15) that $R=\rho g(L-x)+\frac{1}{2} \rho V^{2}$ ?

\section{CONCLUSION}

From above insightful discussions, we can comprehensively conclude that:
1. Herein, we have clearly proved the existence and importance of impact energy loss and gracefully unveiled its crucial role both in so-called incompressible fluid flow dynamics and associated computational fluid dynamics.

2. A considerable amount of impact energy loss could occur in incompressible fluid flow especially in pipe expansion and associated flows.

3. Due to impact effect of high-speed flow ramming into low-speed flow, the energy loss in pipe expansion flow is much greater than corresponding contraction flow with reverse area ratio.

4. Usually, the impact energy loss can easily override the viscous energy loss in incompressible pipe flow with large expansion angle and area ratio.

5. The natural feature of impact energy loss is that it can occur within extremely short time duration while viscous energy loss usually accomplishes in a relatively much longer time period.

6. For so-called incompressible flow, impact energy loss is largely independent of fluid viscosity and can occur in both viscous and inviscid flow as illustrated by the cold/hot water pipe flow with large expansion angle and area ratio.

7. More importantly and surprisingly, the unveiling of the crucial role of impact energy loss for incompressible fluid flow fluently reveals the non-genuineness of Euler equation as a momentum equation due to its incapability at all to accommodate any cases with energy loss. In turn, this non-genuineness unveils the even more stunning role of impact energy loss for triggering a full-scale epoch-making revolution in FD/CFD as deliberated in the related paper with title "The Life and Death of Euler, Bernoulli, Navier-Stokes Equation and Associated CFD for So-called Incompressible Fluid Flow" [10].

8. The intrinsic negligence of impact energy loss is the original major cause for many weird paradoxes and disaster solutions, such as d'Alembert paradox of uniform flow past a circular cylinder in potential flow and associated CFD.

9. The non-genuineness as a momentum equation due to the intrinsic negligence of impact energy loss in deriving process is the original major reason why the seemingly simple-looking Euler equation for incompressible, inviscid flow constitutes one of the most difficult problems in fluid dynamics and CFD to be solved, because the solution may not exist at all.

10. High speed flow ramming into low speed flow in pipe expansion flow is similar to the continuous inelastic impact process in system dynamics with unavoidable impact energy loss. The striking ropelifting example literally demonstrates the existence 
and importance of impact energy loss not only in fluid dynamics but also in related system dynamics, and shows what a disaster solution can incur if one neglects the impact energy loss no matter explicitly or implicitly. Although this standard rope-lifting problem is widely taught in many universities throughout the world, who can believe this kind of absurdity that $R=\rho g(L-x)+\frac{1}{2} \rho V^{2}$ ?

11. In computational fluid dynamics practice for steady incompressible fluid flow, many kinds of sophisticated ad hoc treatments such as artificial viscosity, numerical dissipation, upwinding, artificial compressibility, and so forth are devised awkwardly in an attempt to somehow manipulate a certain amount of disguised energy dissipation which is often largely due to impact.

\section{REFERENCES}

1. Anderson, J.D., Computational Fluid Dynamics, McGraw-Hill, Singapore, p. 145 and p. 243 (1995).

2. Birkhoff, G., Hydrodynamics - a Study in Logic, Fact and Similitude, Princeton University Press, Princeton, NJ (1960).

3. Caughey, D.A. and Hafez, M.M., Frontiers of Computational Fluid Dynamics 1998, World Scientific, Singapore, p. 172 (1998).

4. Deconinck, H. and Koren, B., Euler and Navier-Stokes Solvers Using Multi-Dimensional Upwind Schemes and Multigrid Acceleration, Vieweg and Sohn, Wiesbaden (1997).

5. Gresho, P. M., Sani, R.L., and Engelman, M.S., Incompressible Flow and the Finite Element Method, John Wiley, Chichester, pp. 125-129 (1998).

5. Gallavotti, G., Foundations of Fluid Dynamics, SpringerVerlag, Heidelberg (2002).

7. Habashi, W.G. and Hafez, M.M., Computational Fluid Dynamics Techniques, Gordon and Breach Publishers,
SA, p. 367 (1995).

8. Jiang, B.-N., The Least-Squares Finite Element Method, Springer-Verlag, Heidelberg, p. 241 (1998).

9. Ladyzhenskaya, O.A., The Mathematical Theory of Viscous Incompressible Flow, Gordon and Breach, Science Publishers, p. 1 (1987).

10. Lee, H.J. and Chiang, J.S., "The Life and Death of Euler, Bernoulli, Navier-Stokes Equations and Associated CFD for So-called Incompressible Fluid Flow," anticipated to appear.

11. Lee, H.J., Chang, M.Y., and Chan, J.M., "A Forbidden Zone of No-energy-loss Assumption: The Rope-lifting Problem," The Fifteenth National Conference on Theoretical and Applied Mechanics, Tainan, Taiwan, pp. 1067-1071 (1991).

12. Lomax, H., Pulliam, T.H., and Zingg, D.W., Fundamentals of Computational Fluid Dynamics, Springer-Verlag, Heidelberg, p. 189 (2001).

13. Meriam, J.L. and Kraige, L.G., Engineering Mechanics, Vol. 2, Dynamics, 2nd Ed., John Wiley, New York, p. 279 (1987).

14. Mott, R.L., Applied Fluid Mechanics, Prentice-Hall, London, pp. 272-273 (2000).

15. Munson, B.R., Young, D.F., and Okiishi, T.H., Fundamentals of Fluid Mechanics, 3rd Ed., John Wiley, New York, p. 501 and pp. 586-587 (1998).

16. Patankar, S.V., Numerical Heat Transfer and Fluid Flow, Hemisphere, Washington, D.C. (1980).

17. Roberson, J.A. and Crowe, C.T., Engineering Fluid Mechanics, 2nd Ed., Moughton Mifflin Co., Boston, p. 413 (1980).

18. Turek, S., Efficient Solvers for Incompressible Flow Problems, Springer-Verlag, Berlin, pp. 16-26 (1999).

19. Wendt, J.F., Computational Fluid Dynamics - an Introduction, Springer-Verlag, New York, p. 6 (1992).

20. Wesseling, P., Principles of Computational Fluid Dynamics, Springer-Verlag, Berlin (2001). 